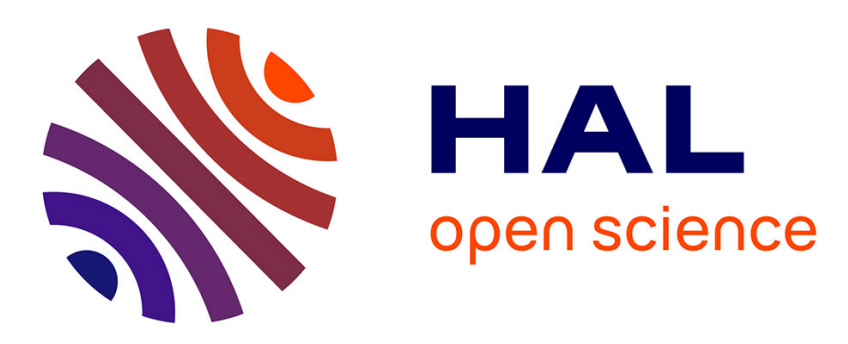

\title{
Origin of frequency difference between damped and sustained modes in vibrating wire sensors
}

Bingqing Mei, Jérôme Lucas, Stéphane Holé, Isabelle Lamarque, Norbert

Cheron

\section{To cite this version:}

Bingqing Mei, Jérôme Lucas, Stéphane Holé, Isabelle Lamarque, Norbert Cheron. Origin of frequency difference between damped and sustained modes in vibrating wire sensors. Sensors and Actuators A: Physical , 2016, 241, pp.66-73. 10.1016/j.sna.2016.02.005 . hal-01314574

\section{HAL Id: hal-01314574 https://hal.sorbonne-universite.fr/hal-01314574}

Submitted on 11 May 2016

HAL is a multi-disciplinary open access archive for the deposit and dissemination of scientific research documents, whether they are published or not. The documents may come from teaching and research institutions in France or abroad, or from public or private research centers.
L'archive ouverte pluridisciplinaire HAL, est destinée au dépôt et à la diffusion de documents scientifiques de niveau recherche, publiés ou non, émanant des établissements d'enseignement et de recherche français ou étrangers, des laboratoires publics ou privés. 


\title{
Origin of frequency difference between damped and sustained modes in vibrating wire sensors
}

\author{
Bingqing Mei ${ }^{1}$, Jérôme Lucas ${ }^{1}$, Stéphane Holé ${ }^{1}$, Isabelle Lamarque ${ }^{2}$, Norbert Cheron ${ }^{2}$ \\ ${ }^{1}$ PSL Research University, ESPCI-ParisTech, Sorbonne Universités, UMPC Univ Paris 06, CNRS, LPEM \\ UMR 8213, 10 rue Vauquelin, 75005 Paris, France \\ ${ }^{2}$ Géo-Instrumentation, 128 bis Av. Jean-Jaurès, 94200 Ivry sur Seine, France \\ Corresponding author: Stéphane Holé \\ e-mail: stephane.hole@espci.fr \\ telephone: (33)(0)1 40794563
}

\begin{abstract}
Vibrating wire sensors can operate in two modes, damped mode and sustained mode. In practice, the resonant frequency measured in sustained mode is higher than the one measured in damped mode. In this paper, the theoretical analysis indicates that average magnetic force and coil coupling are responsible for the frequency difference. This prediction is experimentally confirmed by measuring the resonant frequencies for different values of the two parameters. It is shown how to correct measurements in order to obtain the same results for the two modes.
\end{abstract}

Keywords: Vibrating wire sensor; Damped mode; Sustained mode; Frequency difference; Magnetic force; Coil coupling.

\section{Introduction}

Vibrating wire sensors are widely used to monitor deformations of large civil structures such as dams, bridges and nuclear power plants [1]. This type of sensors is based on a stainless steel wire which is set into vibration by an excitation coil and this vibration is detected by the same coil or an additional coil depending on the measurement mode [2]. The wire is tightly stretched between two anchors installed in the monitored structure, as shown in Figure 1. Small relative movements of the two anchors alter the mechanical tension of the wire and hence its resonant frequency [3]. Therefore the deformation of the structure can be determined by measuring the resulting change in the resonant frequency of the wire [4]. 


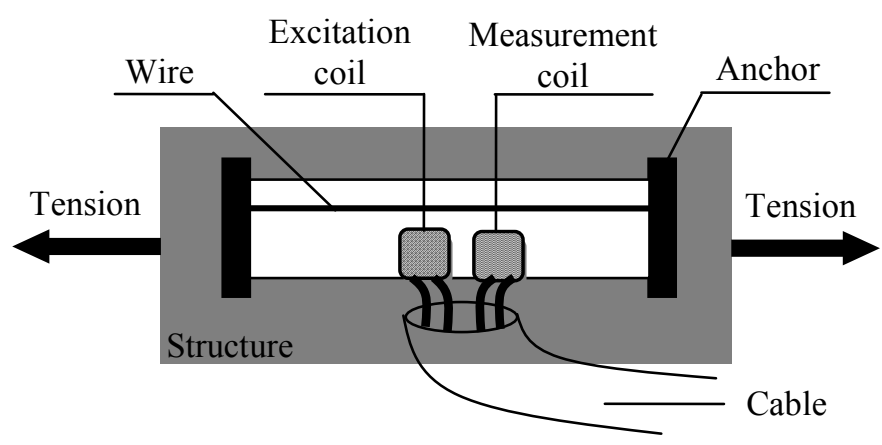

Fig. 1 Vibrating wire sensor installed in the monitored structure

There are two modes of operation: damped mode that corresponds to a measurement when the wire is freely vibrating after a pulsed excitation, and sustained mode that corresponds to a measurement when the wire is continuously excited at its resonant frequency. Historically, the sensors operating in damped mode have been in use since about 1930 [5], and those operating in sustained mode have been adopted around 1960 [6]. It is commonly considered that the resonant frequency of the vibrating wire depends primarily on the wire properties (length, density and tension), and very little on the operation mode of the sensors. However, this is not exactly true. According to various experimental tests $[7,8]$, the resonant frequency measured in damped mode differs from the one measured in sustained mode by $0.1-0.3 \%$. Since the vibrating wire sensors are expected to be accurate at $0.1 \%$, this frequency difference is far from being negligible. Moreover this makes it difficult to compare the measurements obtained in one mode and another. To properly interpret the measurements, one needs to fully understand the sensor signals, thus finding out what causes the frequency difference and how to correct it. In the case of two-coil sensors, if one of the coils fails, it is necessary to switch from sustained mode to damped mode [9]. In that case, it is very important to compensate the frequency difference.

In this paper, firstly the sensor operation in damped and sustained modes is analyzed in order to find the possible parameters that may affect the resonant frequency. Secondly, the influence of the relevant parameters on the resonant frequency is experimentally investigated in the two operating modes.

\section{Relevant parameters of resonant frequency}

The operation of vibrating wire sensors relies on the wire excitation and frequency measurement. Both can be accomplished using magneto-mechanical coupling. There are two modes of operation commonly used today: damped mode corresponding to pulsed excitation and sustained mode corresponding to continuous excitation [10]. 


\subsection{Damped mode}

The damped mode consists in giving the wire an initial displacement and then measuring the frequency of the damped free vibration [11]. The operating procedure in damped mode is illustrated in Figure 2. Initially a single pulse or a burst is applied to the excitation coil [12], thereby creating a magnetic field that excites the wire during a short period of time (Figure 2a). Then the wire vibrates until the vibration is completely damped, within seconds. The damped vibration induces, due to the remaining magnetic field, for instance due to the magnetization of the coil core produced by the excitation, a voltage in the same coil or a second coil (Figure $2 \mathrm{~b}$ ). The frequency and the temporal evolution of this voltage are identical to those of the wire vibration.

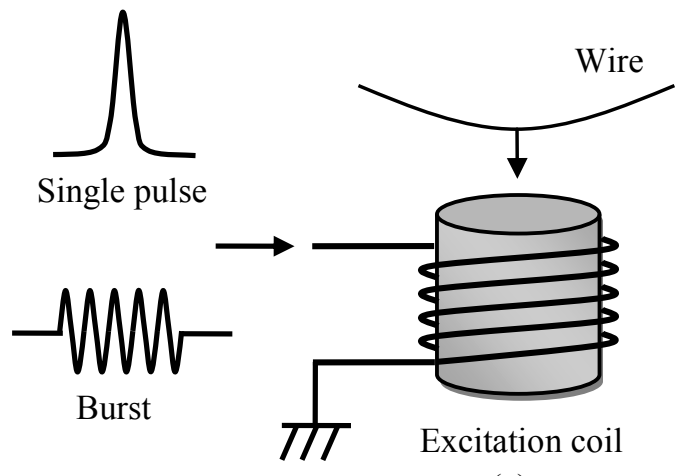

(a)

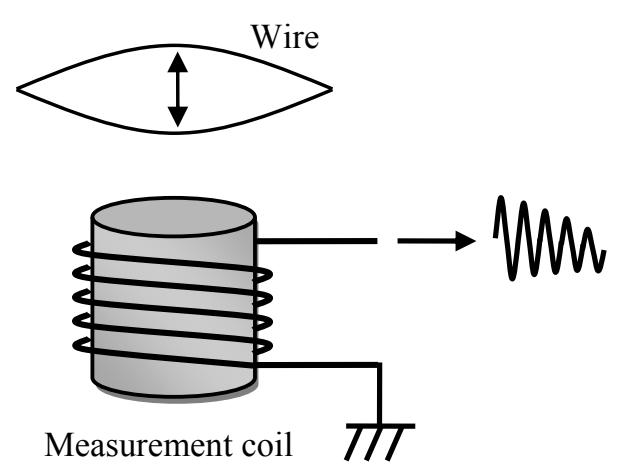

(b)

Fig. 2 Damped mode. (a) Pulsed excitation. (b) Measurement of the damped vibration

After the pulsed excitation, the absence of external force (magnetic force) allows the wire to undergo damped free vibration due to the mechanical tension in the wire. Consider a uniform stretched wire of mass density $\rho$, cross sectional area $A$, under constant tension $T$. For the sake of simplicity, the gravity and the bending stiffness of the wire are always ignored. The equation of motion of the wire undergoing free vibration can be written as

$$
\rho A \frac{\partial^{2} u_{y}}{\partial t^{2}}=T \frac{\partial^{2} u_{y}}{\partial x^{2}}
$$

where $u_{y}(x, t)$ is the transverse vibration of the wire at position $x$ at time $t$, Assuming that the ends of the wire of length $L$ are fixed at $x=0$ and $x=L$, one has to impose the boundary conditions: $u_{y}(0, t)=$ $u_{y}(L, t)=0$. Applying the boundary conditions to equation (1) leads to the expression of the fundamental natural frequency [13]: 


$$
f_{0}=\frac{1}{2 L} \sqrt{\frac{T}{\rho A}}
$$

In the damped vibration, the actual frequency measured by the coil is slightly lower than the natural frequency $f_{0}$ because of damping influence. The apparent damped frequency $f_{a}$ related to the damping ratio $\zeta$ is given by

$$
f_{a}=f_{0} \sqrt{1-\zeta^{2}}
$$

\subsection{Sustained mode}

The sustained mode consists in maintaining the wire at resonance and meanwhile measuring the resonant frequency [14]. In this mode, it is necessary to use two coils, one to continuously excite the wire and the other to simultaneously measure the frequency of the vibration. The operating procedure in sustained mode is illustrated in Figure 3. The first coil is supplied by a sinusoidal voltage at the resonant frequency of the wire. The continuous excitation generates a sinusoidally varying magnetic field which maintains the wire in resonant vibration. The wire displacement induces an alternating voltage in the second coil. This induced voltage has the same frequency as the wire vibration.
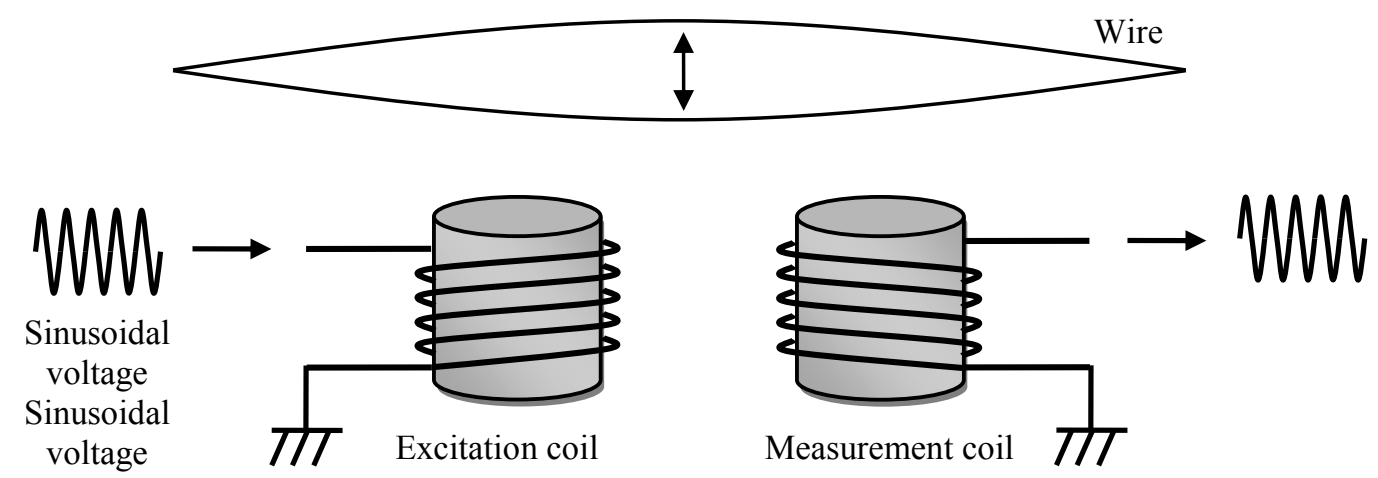

Fig. 3 Sustained mode. Excitation and measurement are continuous and simultaneous

In sustained mode, the excitation always exists. Thus the magnetic force continues to act on the wire periodically, and the wire is forced to vibrate at the excitation frequency. In theory, the resonance occurs when the forced frequency is equal to the natural frequency of the wire [15]. However, in practice, we have to consider two additional parameters: average magnetic force and mutual coupling between coils. 


\subsection{Effect of average magnetic force}

The current passing through the excitation coil creates a magnetic field $H$ around the wire. Since the stainless steel wire has a magnetic permeability greater than that of air $\left(\mu_{0}=4 \pi \times 10^{-7} \mathrm{H} / \mathrm{m}\right), H$ is guided along the wire. As a consequence, the magnetic field repartition is such that $H$ is relatively large just under the wire but much smaller just above and inside the wire. This results in a magnetic stress $\Gamma_{\text {mag }}=1 / 2 \mu_{0} H^{2}[16]$ which is larger on the bottom than on the top of the wire, as shown in Figure 4. Thus the wire is bent towards the coil.

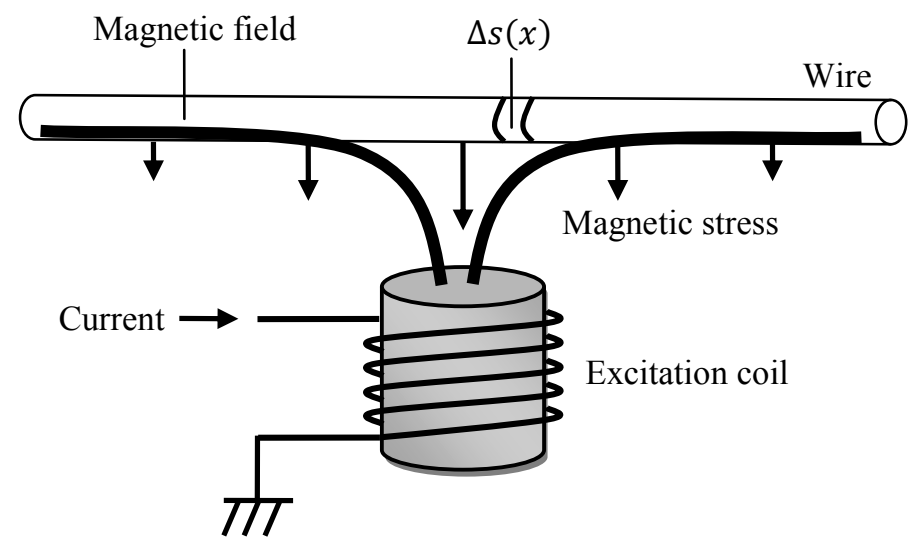

Fig. 4 Excitation of the wire with magnetic force

In sustained mode, equation (1) then becomes

$$
\rho A \frac{\partial^{2} u_{y}}{\partial t^{2}}=T \frac{\partial^{2} u_{y}}{\partial x^{2}}+\int_{\Delta s(x)} \frac{1}{2} \mu_{0} H^{2} d \vec{s}
$$

where $\Delta s(x)$ is the surface of an elementary portion $d x$ of the wire at position $x$. As the magnetic field $H$ depends on many geometrical parameters, equation (4) is difficult to resolve most of the time. Nevertheless, an approximate expression of the resonant frequency can be deducted by investigating the increase in the mechanical tension resulting from the magnetic force. During the free vibration in damped mode, no magnetic force acts on the wire, therefore the wire lies straight and its resonant frequency depends only on the mechanical tension $T$, as shown in Figure 5a. During the forced vibration in sustained mode, the wire is subjected to the tension $T$ as in damped mode and a magnetic force $F_{m a g}$ that pulls the wire perpendicularly towards the coil, as shown in Figure 5b. The magnetic force produces an elongation of the wire $\delta L$ and thus a variation of the wire tension $\delta T$. When $F_{m a g}$ is applied perpendicularly to the wire at its center, one obtains $\delta L \approx 2 y_{0}^{2} / L$, where $y_{0}$ is the wire transverse displacement. With Hooke's law, the variation of the wire tension is 


$$
\delta T=E A \frac{\delta L}{L}=\frac{2 E A y_{0}^{2}}{L^{2}}
$$

where $E$ is the wire Young's modulus. Because $\delta T \ll T$, the transverse displacement $y_{0}$ can be deduced from the average magnetic force $\overline{F_{m a g}}$ by $\overline{F_{m a g}}=2 T \sin \theta \approx 4 y_{0} T / L$, thus $y_{0} \approx \overline{F_{m a g}} L / 4 T$. According to expression (2), the increase in tension $\delta T$ leads to an increase in resonant frequency. Moreover, as the magnetic force is proportional to the power of the excitation, an increase in excitation amplitude (AC component) or in offset (DC component) should cause an increase in resonant frequency.

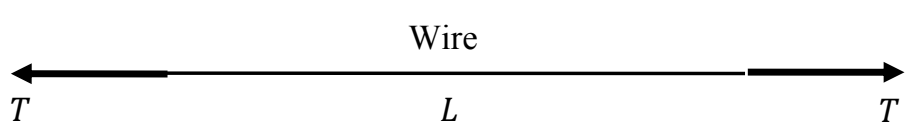

(a)

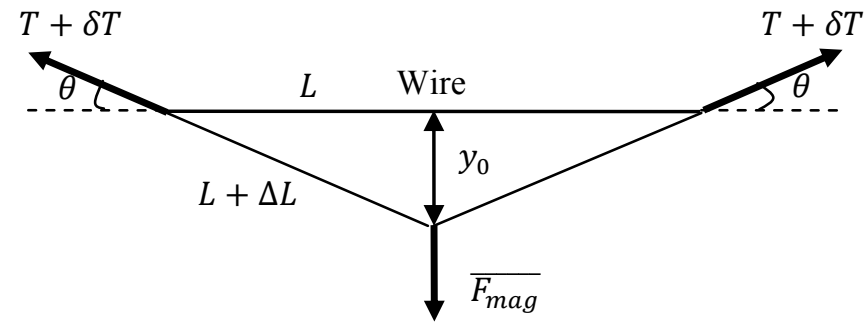

(b)

Fig. 5 Effects of the forces involved in the wire vibration in (a) damped mode and (b) sustained mode

As an illustration, let us consider a perfectly flexible wire of length $L_{w}=110 \mathrm{~mm}$ and radius $r_{w}=115 \mu \mathrm{m}$, stretched under tension $T=15 \mathrm{~N}$. The material properties of stainless steel wire are: masse density $\rho=$ $7800 \mathrm{~kg} / \mathrm{m}^{3}$ and Young's modulus $E=210 \mathrm{GPa}$. The excitation coil of radius $r_{b}=1.5 \mathrm{~mm}$, having 200000 turns per meter and a high permeability core of $\mu_{r}=1000$, is located $2 \mathrm{~mm}$ above the center of the wire. When a current of $I=50 \mathrm{~mA}$ is passing through the coil, the magnetic field at the wire is approximately $5 \times 10^{5} \mathrm{~A} / \mathrm{m}$. Considering also a surface on the wire regarding the coil of $s=0.5 \mathrm{~mm}^{2}$, one obtains an average magnetic force applied at the wire midpoint $\overline{F_{m a g}}=s \mu_{0} H^{2} / 2=0.08 \mathrm{~N}$. According to expressions (2) and (5), the wire tension is increased by $0.05 \%$ and thus the resonant frequency is increased by about 1 $\mathrm{Hz}$.

\subsection{Effect of coil coupling}

Two couplings must be taken into account in the measurement system. Firstly, the wire vibration produces a modulation of the remaining magnetic field in the vicinity of the measurement coil. Therefore a coupling takes place between the wire and the measurement coil (wire-coil coupling). Secondly, the continuous sinusoidal voltage in the excitation coil produces an induced voltage in the measurement coil because of the mutual inductance. Thus a coupling appears between the two coils (coil-coil coupling). The wire-coil 
coupling exists in both damped and sustained modes, whereas the coil-coil coupling exists only in sustained mode.

In damped mode, after an initial pulsed excitation, the remaining magnetic field along with the wire vibration is the only cause of the variation of the magnetic flux $\phi_{s}$ in the measurement coil and thus of the voltage $V_{m}$ induced in the measurement coil. The equivalent circuit includes a magnetic source $\partial \phi_{S} / \partial t$ and the selfinductance $L$ of the coil which presents an internal resistance $R$. One has $V_{m}=\partial \phi_{s} / \partial t+\partial\left(L i_{m}\right) / \partial t+R i_{m}$, which corresponds to the equivalent circuit of the measurement set up shown in Figure 6a. Since the magnetic source $\phi_{s}$ is driven by the wire resonance, it depends on frequency as a second order system. This resonance can be modeled around the wire resonant frequency as a parallel RLC circuit excited by a constant amplitude source $\phi_{\mathrm{s} 0}$. The equivalent circuit of Figure 6a becomes the one of Figure $6 \mathrm{~b}$.

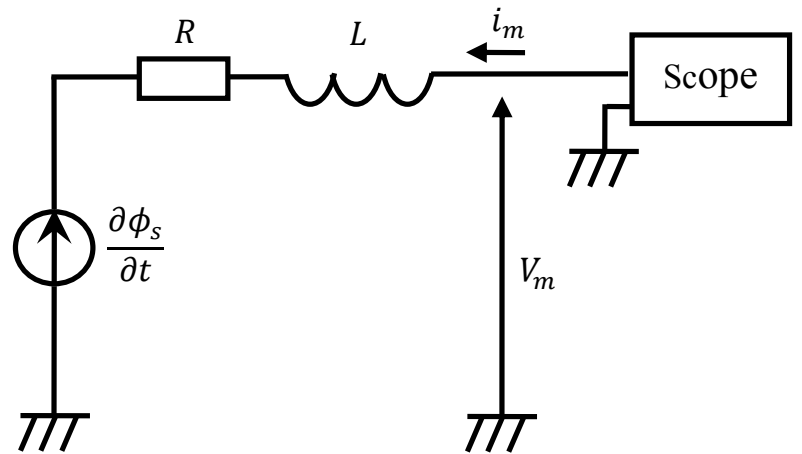

(a)

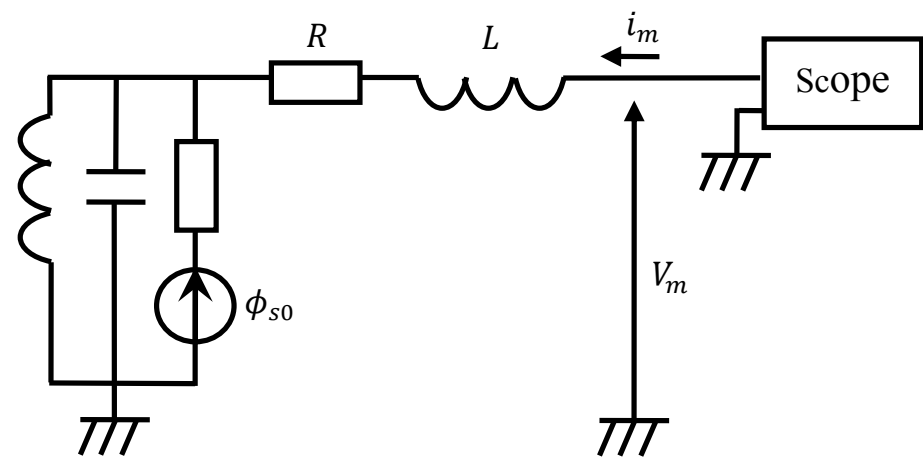

(b)

Fig. 6 Equivalent circuit of vibrating wire sensor in damped mode

In sustained mode, the signal is due to the remaining magnetic field along with the wire vibration, like in damped mode, and to the mutual coupling between the two coils. Accordingly the voltage $V_{m}$ across the measurement coil is $V_{m}=\partial \phi_{s} / \partial t+\partial\left(L i_{m}+M i_{e}\right) / \partial t+R i_{m}$ where $M$ is the mutual inductance between the coils. The equivalent circuit corresponding to this set up is presented in Figure 7a. As in the former case, $\phi_{s}$ depends on frequency which can be modeled around the wire resonant frequency as a parallel RLC circuit. One obtains the equivalent circuit of Figure $7 \mathrm{~b}$. It can be seen that the excitation $V_{e}$ contributes through $M$ to the measured signal and thus shifts the resonant frequency. 


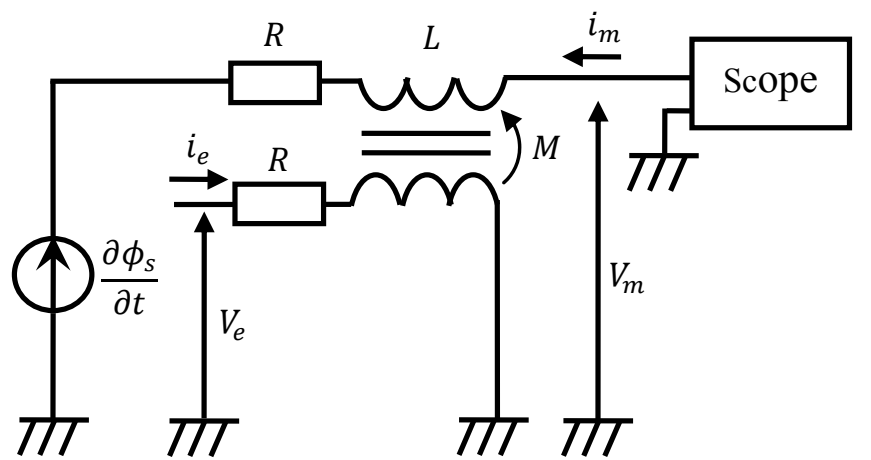

(a)

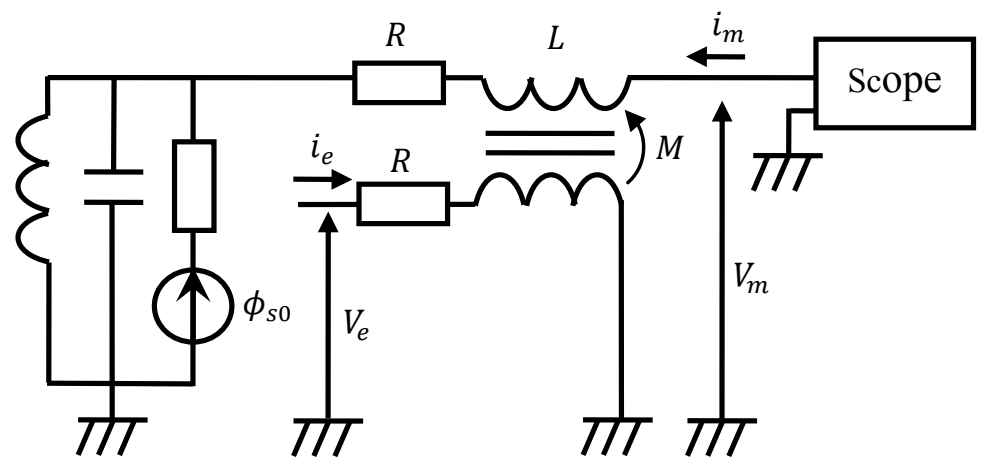

(b)

Fig. 7 Equivalent circuit of vibrating wire sensor in sustained mode

\section{Experimental results}

\subsection{Experimental set up}

An open sensor has been realized instead of industrial closed sensor for creating a test environment. Figure 8 shows the experimental set up. A stainless steel wire is stretched between two arms. Two equal weights are respectively placed on each arm to give the wire a controlled mechanical tension. Two coils, positioned near the wire, can be shifted along its axis. The excitation coil is connected to a signal generator, and the measurement coil is connected to an oscilloscope. This makes it possible to control precisely the excitation of the wire. The experiments aim to demonstrate that average magnetic force and coil coupling are responsible for the frequency difference between two modes. For that purpose, firstly the resonant frequencies are measured in damped and sustained modes. Then the effects of average magnetic force and coil coupling are respectively investigated.

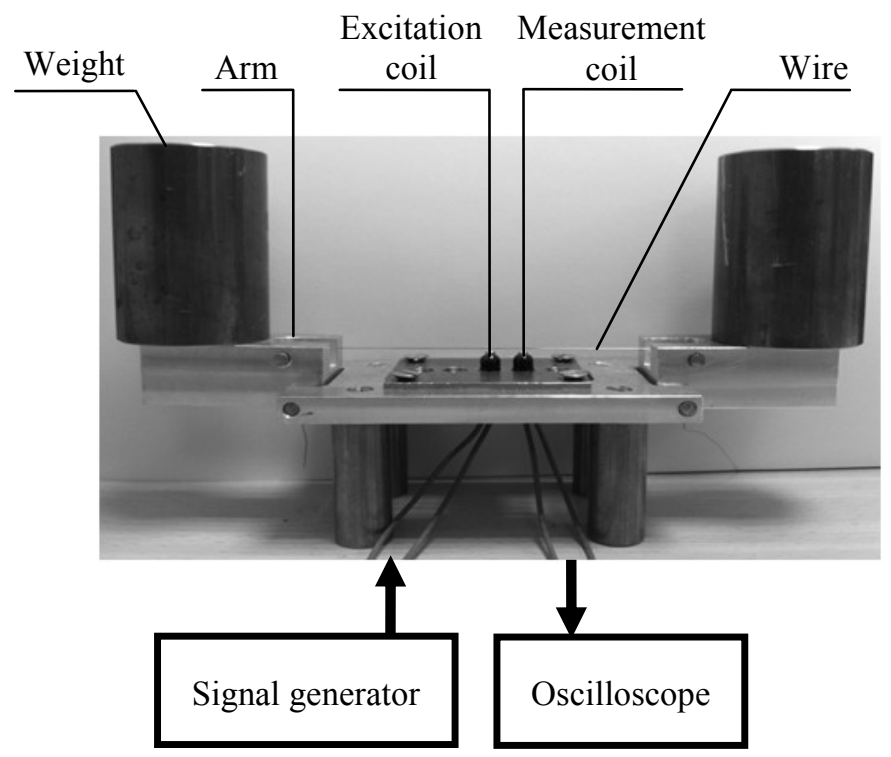

Fig. 8 Experimental set up for studying the wire vibration in damped and sustained mode 


\subsection{Results of resonant frequency}

In damped mode, at first a burst of 200 cycles is sent to the excitation coil. Each cycle corresponds to a sinusoidal voltage of $1-\mathrm{kHz}$ frequency and $1.5-\mathrm{V}$ amplitude, as shown in Figure 9a. After the pulsed excitation, the output signal of the measurement coil is displayed on the oscilloscope, as shown in Figure $9 \mathrm{~b}$. This signal is measured after the pulsed excitation and a dead time of approximately $50 \mathrm{~ms}$, thus it is only the free response of the wire. The wire response appears as a damped oscillation whose amplitude decreases exponentially with time. The rate of decrease in amplitude is represented by the damping factor $\zeta$, which is equal to $2 \times 10^{-4}$. Since a vibrating wire sensor has various resonant frequencies which are not integer multiples of the fundamental resonant frequency due to the bending stiffness of the wire [17], the fast Fourier transform algorithm is used for spectral analysis. The FFT time window is set to $4.8 \mathrm{~s}$ (only $2.5 \mathrm{~s}$ shown in Figure $9 \mathrm{~b}$ for the sake of clarity) so that the frequency accuracy is $0.21 \mathrm{~Hz}$. This accuracy can be increased using interpolated FFT. Here all frequencies determined using FFT are accurate at $\pm 0.21 \mathrm{~Hz}$. The frequency spectrum of the wire response is shown in Figure 10. The highest peak located at $999.64 \mathrm{~Hz}$ represents the fundamental frequency of the damped free vibration. During the wire vibration, the distance between the measurement coil and the wire changes within each period of vibration, and thus the induced signal has not the same amplitude when the wire is close to the measurement coil or far from the measurement coil. The non-linearity of the measurement system adds obviously harmonics which are perfectly integer multiples of the fundamental resonant frequency [18], but we are only interested in the fundamental resonant frequency in this study. Substituting $f_{a}=999.64 \mathrm{~Hz}$ and $\zeta=2 \times 10^{-4}$ into equation (3), one obtains $f_{0}=999.64 \mathrm{~Hz}$. Since the damping factor is very small, the damped frequency is almost equal to the natural frequency of the vibrating wire.

(a)

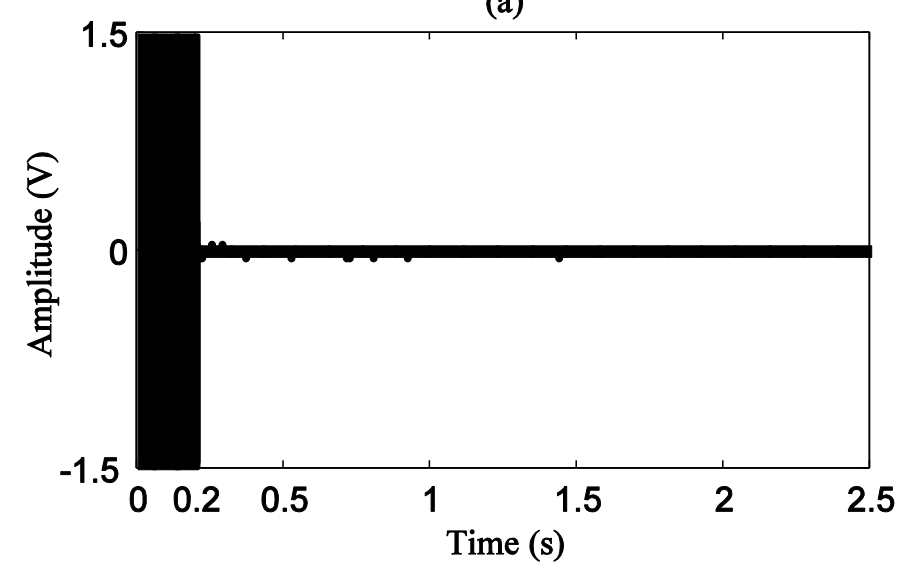

(b)

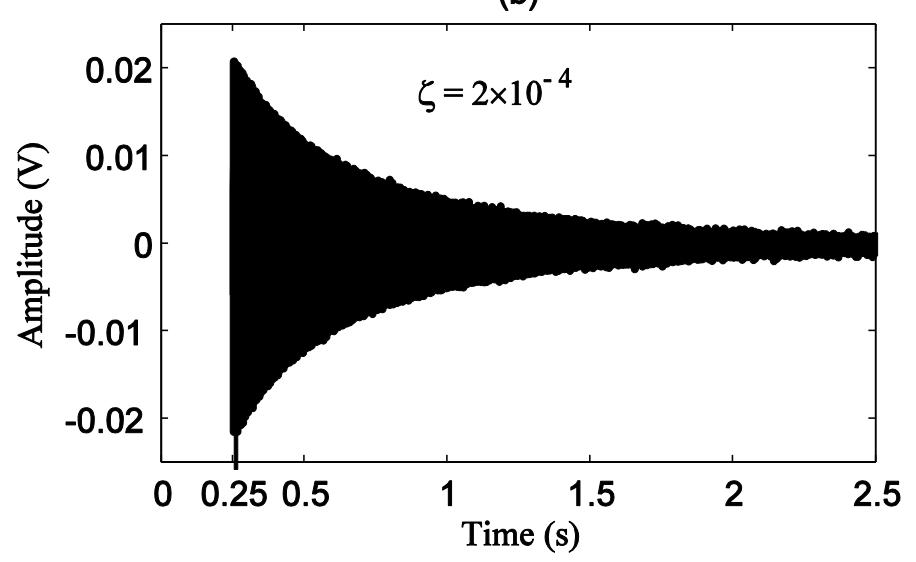

Fig. 9 Damped mode (a) pulsed excitation and (b) free vibration of the wire 


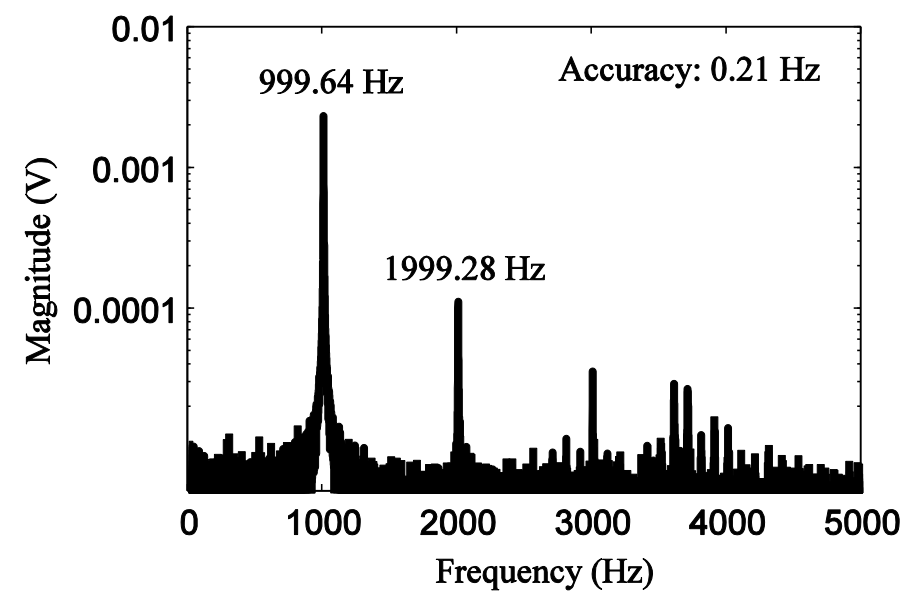

Fig. 10 Frequency spectrum of the wire response in damped mode

In sustained mode, the signal generator continuously excites the wire via the excitation coil, and meanwhile the forced vibration of the wire is detected by the measurement coil. In order to find the fundamental resonant frequency, we set the frequency of the signal generator at the natural frequency $999.64 \mathrm{~Hz}$. The frequency is slowly adjusted in $0.01 \mathrm{~Hz}$ steps until the output signal of the measurement coil reaches its maximum. Therefore the frequency accuracy when turning the signal generator is $\pm 0.01 \mathrm{~Hz}$. The maximal amplitude is reached at $1001.93 \mathrm{~Hz}$. The dashed curve in Figure 11 represents the excitation signal which is a sinusoidal voltage of $1.5-\mathrm{V}$ amplitude, and the solid curve in the same figure denotes the measured signal which is amplified 5 times. Figure 12 gives the frequency spectrum of the wire vibration in sustained mode. Here again the small harmonic at twice the first resonant frequency is due to the non-linearity of the measurement system. Compared to the natural resonant frequency measured in damped mode (see Figure 10), the resonant frequency measured in sustained mode is increased by $2.29 \pm 0.22 \mathrm{~Hz}$.

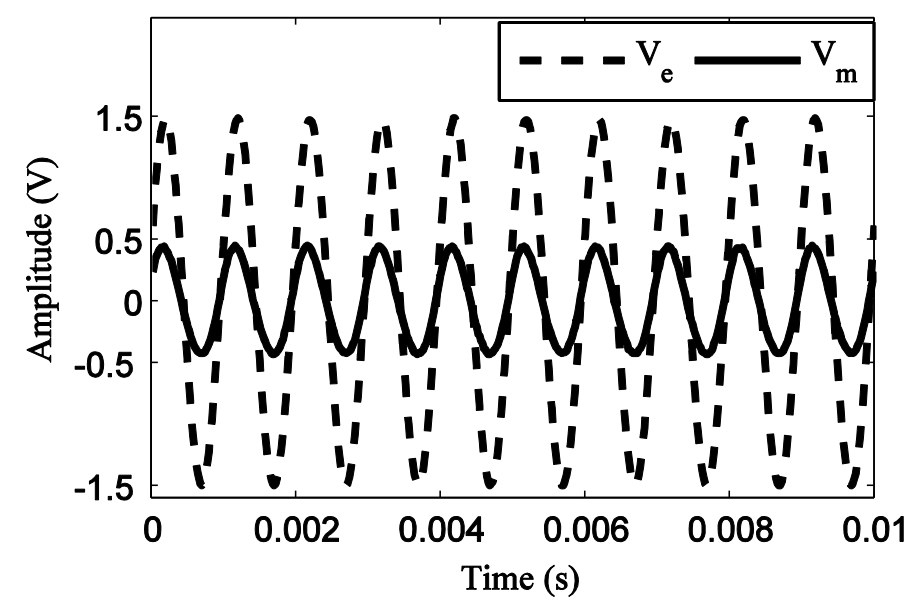

Fig. 11 Excitation signal and measurement signal in sustained mode 


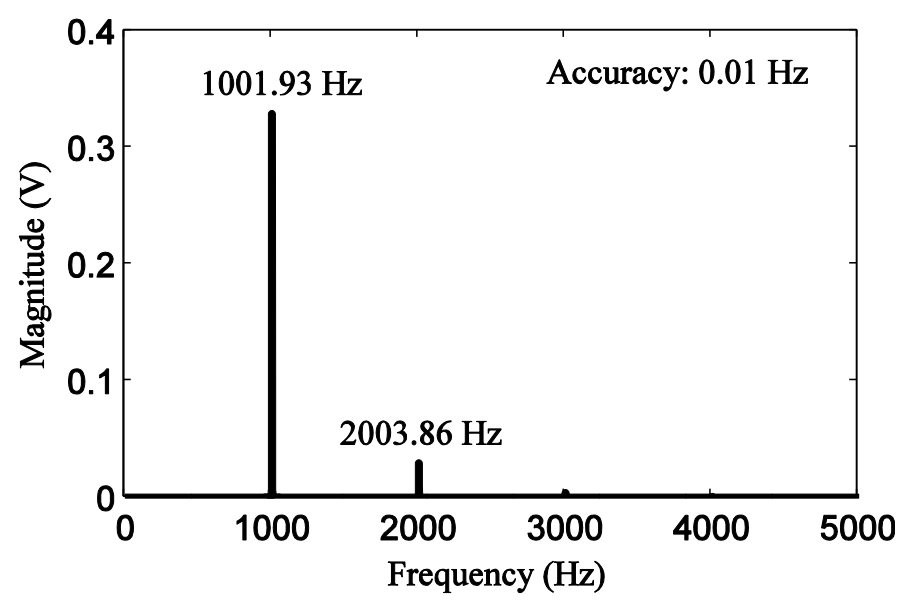

Fig. 12 Frequency spectrum of the wire response in sustained mode

\subsection{Average magnetic force study}

The effect of average magnetic force is studied by varying the excitation amplitude. Figure 13a and 13b show respectively the resonant frequency measured in damped and sustained modes under different amplitudes of excitation. To keep the same initial conditions of the wire vibration, each change of the excitation amplitude is independent of all previous ones. For example, after finding the resonant frequency under the first excitation of amplitude $0.75 \mathrm{~V}$, the generator output is switched off in order to make the wire go back into rest. When the wire is at rest, the generator output is set to $1.5 \mathrm{~V}$ and is switched on. Then we search for the resonant frequency as previously done in the case of the first excitation. In addition, the transverse displacement of the wire is never very large $(<0.2 \mathrm{~mm})$ with respect to the wire diameter $(\approx 0.2 \mathrm{~mm})$. This allows us to avoid non-linear vibration. During our measurements, non-linear vibrations were never observed. All the observed harmonics are caused by the non-linearity of the measurement system. It can be seen that the excitation amplitude does not influence the natural resonant frequency in damped mode (Figure 13a), whereas the resonant frequency increases with the excitation amplitude in sustained mode. Above $6 \mathrm{~V}$, the wire touches one of the coils, thereby causing a slight step visible in both modes (Figure 13b). The experimental results are in accordance with the theoretical prediction of section 2.3 since, as expected, the resonant frequency varies with the excitation amplitude in sustained mode but not in damped mode. A linear curve fitting is performed to predict the resonant frequency at $0 \mathrm{~V}$, as indicated by the dashed line. When the excitation amplitude decreases from $1.5 \mathrm{~V}$, which corresponds to the excitation level in the experiment of Figure 11 , to $0 \mathrm{~V}$, the resulting reduction in resonant frequency is $1001.93-1000.81=1.12 \mathrm{~Hz}$. The $95 \%$ confidence interval for the predicted difference is $1.12 \pm 0.09 \mathrm{~Hz}$. Notice that this frequency difference is more than 10 times the expected accuracy of a vibrating wire sensor which is typically of $0.1 \mathrm{~Hz}$. 
(a)

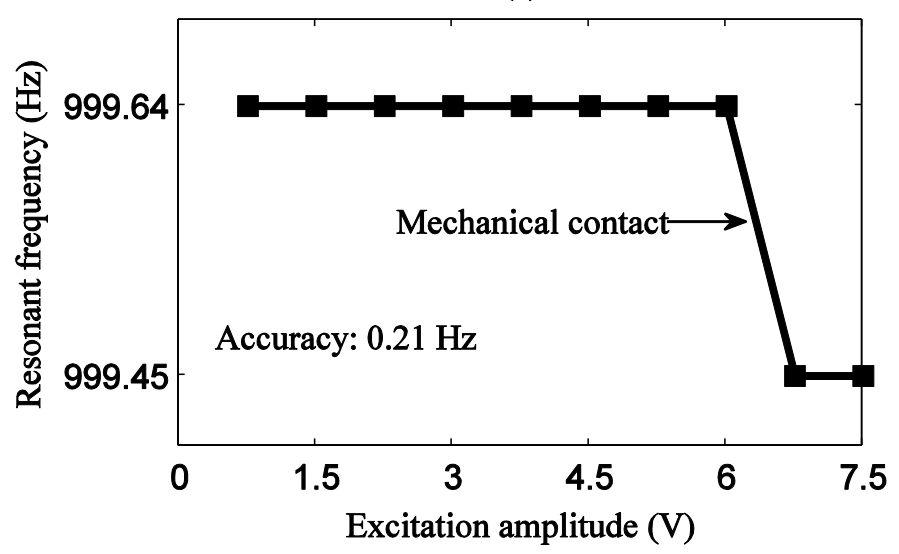

(b)

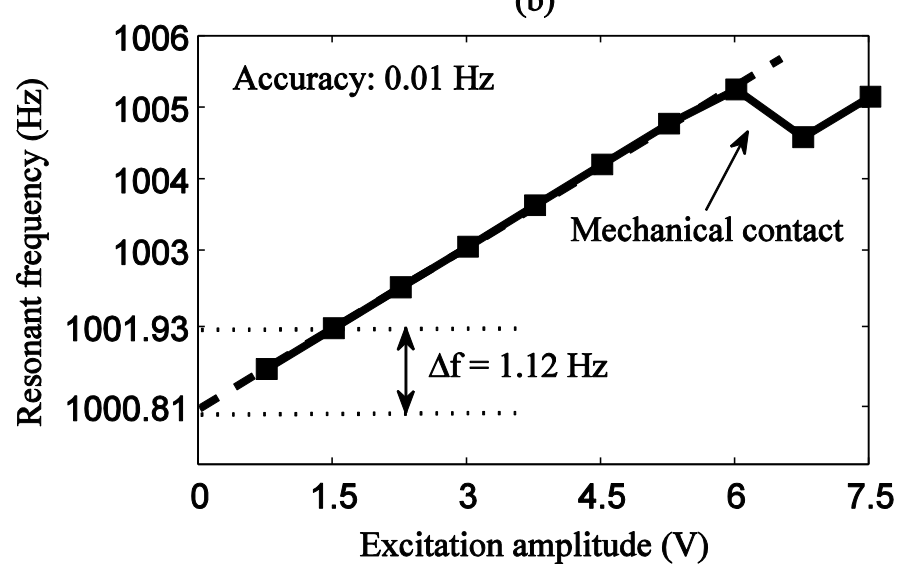

Fig. 13 Relationship between resonant frequency and excitation amplitude in (a) damped mode and (b) sustained mode in the case of a resonant frequency of about $1 \mathrm{kHz}$

In order to confirm that the effects observed above are independent of that specific resonant frequency, the measurements are remade for another working frequency. By adjusting the wire tension, the resonant frequency of the experimental system is now set to $1207.51 \mathrm{~Hz}$ in damped mode (Figure 14a). The difference between the resonant frequency under excitation of $1.5 \mathrm{~V}$ amplitude and the estimated resonant frequency under excitation of $0 \mathrm{~V}$ amplitude is $1209.45-1208.47=0.98 \mathrm{~Hz}$ (Figure 14b). The 95\% confidence interval for the predicted difference is $0.98 \pm 0.11 \mathrm{~Hz}$. Notice that this frequency difference is also more than 10 times the expected accuracy of a vibrating wire sensor.

In order to mimic the effect of a mean magnetic force, another experiment is performed in which the wire is excited by a sinusoidal voltage with constant amplitude superimposed to a variable offset voltage. For a constant excitation amplitude of $1.5 \mathrm{~V}$, the resonant frequency measured in sustained mode under different offset voltages is shown in Figure 15 for a resonant frequency of about $1 \mathrm{kHz}$ or $1.2 \mathrm{kHz}$. Since there is no coupling at $0 \mathrm{~Hz}(\mathrm{DC})$, the results indicate that the frequency difference observed in Figures $13 \mathrm{~b}$ and $14 \mathrm{~b}$ is attributed only to the average magnetic force due to the excitation. As expected the average magnetic force applied to the wire modifies its tension and thus the wire resonant frequency. 
(a)

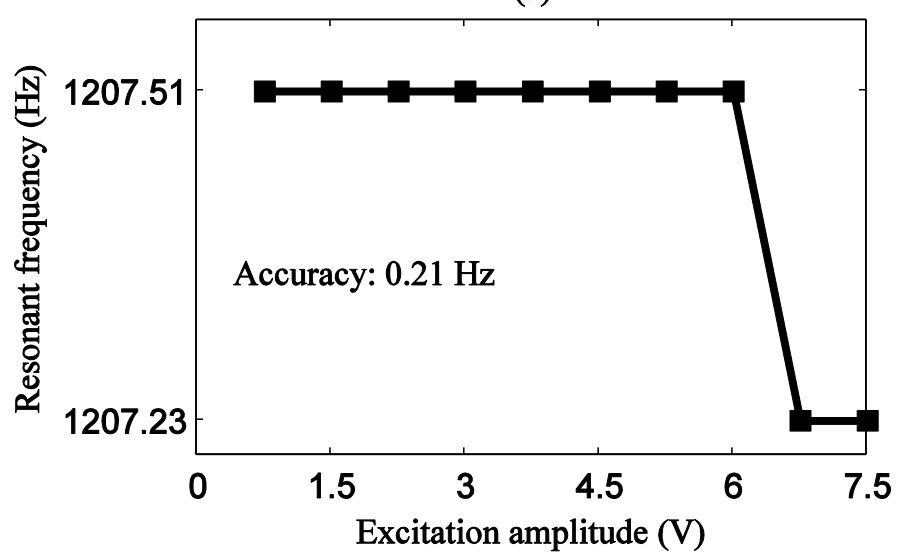

(b)

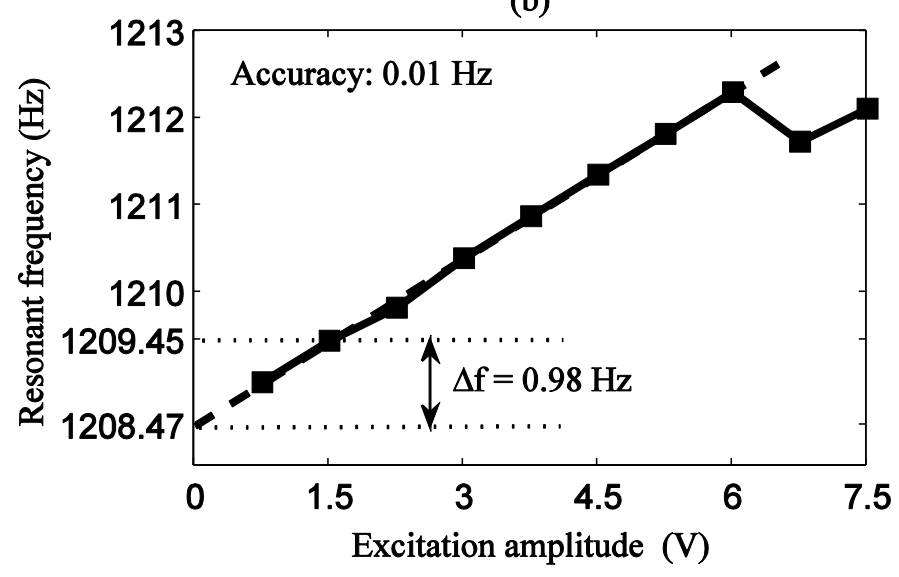

Fig. 14 Relationship between resonant frequency and excitation amplitude in (a) damped mode and (b) sustained mode in the case of resonant frequency of about $1.2 \mathrm{kHz}$

(a)

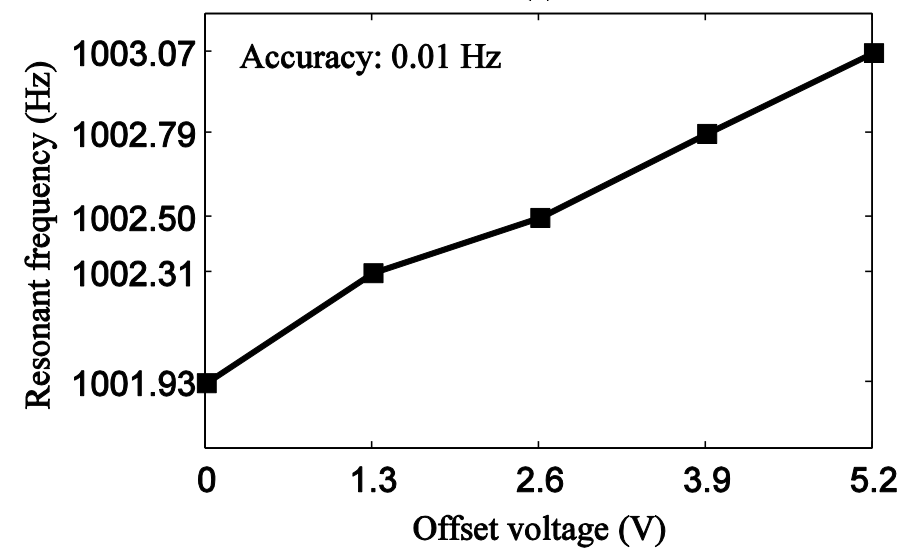

(b)

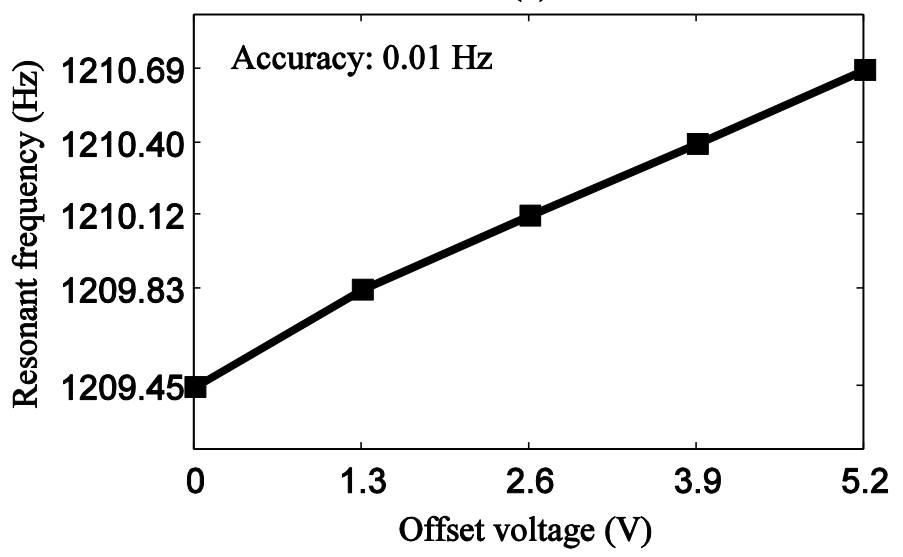

Fig. 15 Relationship between resonant frequency and offset voltage in the case of (a) resonant frequency of about $1 \mathrm{kHz}$ and (b) resonant frequency of about $1.2 \mathrm{kHz}$

\subsection{Coil coupling study}

To study the influence of coil coupling in sustained mode, one first needs to determine the mutual inductance between the two coils. Then it is possible to subtract the induced voltage associated with the coil-coil coupling, and thus to calculate the induced voltage associated with only the wire-coil coupling. It follows that

$$
V_{0}=V_{m e s}(t-\tau)-\alpha V_{e x c}(t)
$$

where $V_{\text {exc }}$ is the excitation signal, $V_{\text {mes }}$ is the measurement signal and $V_{0}$ is the induced voltage without the influence of coil coupling. The amplitude ratio $\alpha$ and the time delay $\tau$ are characteristic of the coil coupling and can vary with frequency. In order to plot the resonance curve of the vibrating wire, an experiment is carried out by changing the frequency of the signal generator from 950 to $1050 \mathrm{~Hz}$ in steps of $1 \mathrm{~Hz}$. At $f_{1}=950 \mathrm{~Hz}$, the excitation frequency is too far from the resonant frequency $(1001.93 \mathrm{~Hz})$ to result in any 
significant wire response. Since the wire is at rest, only the coupling exists between the coils. The time delay $\tau$ between the signals in both coils is directly measured, and by setting $V_{0}$ in equation (6) to zero, one obtains $\tau_{1}=8.47 \times 10^{-5} \mathrm{~s}$ and $\alpha_{1}=0.0355$. A similar situation occurs at $f_{2}=1050 \mathrm{~Hz}$, and the two coefficients are calculated in the same way. One obtains $\tau_{2}=6.77 \times 10^{-5} \mathrm{~s}$ and $\alpha_{2}=0.0357$. Assuming that $\alpha$ and $\tau$ vary slowly with frequency, the following linear interpolation is used to estimate $\alpha$ and $\tau$ at any frequency between $950 \mathrm{~Hz}$ and $1050 \mathrm{~Hz}$ :

$$
\left\{\begin{array}{c}
\alpha=\alpha_{1}+\left(\alpha_{2}-\alpha_{1}\right) \frac{f-f_{1}}{f_{2}-f_{1}} \\
\tau=\tau_{1}+\left(\tau_{2}-\tau_{1}\right) \frac{f-f_{1}}{f_{2}-f_{1}}
\end{array}\right.
$$

At the resonant frequency, one obtains $\tau \approx 7.58 \times 10^{-5} \mathrm{~s}$ and $\alpha \approx 0.0356$. During this process, the amplitude of $V_{\text {exc }}$ is fixed at $1.5 \mathrm{~V}$. The amplitude of $V_{\text {mes }}$ and $V_{0}$ at different excitation frequencies are presented by dashed and solid curves, respectively, in Figure 16. The difference between the two curves corresponds to the elimination of the mutually induced voltage. It is clear that the amplitude of the measured signal in sustained mode depends not only on the wire vibration but also on the mutual coupling between coils.

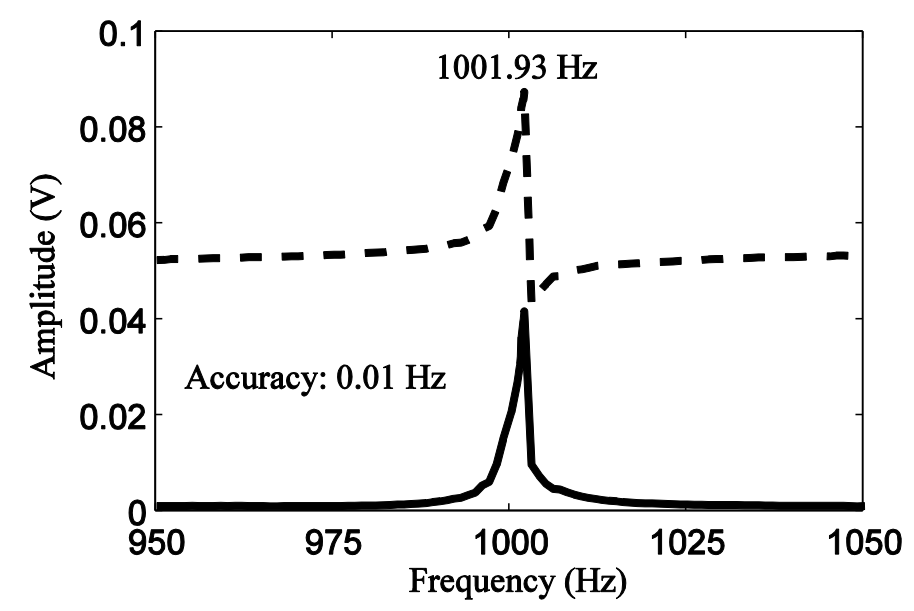

Fig. 16 Resonance curves of the vibrating wire $\left(V_{\text {exc }}=1.5 \mathrm{~V}\right.$ and $\alpha=0.0356$ at resonance $)$

The mutual coupling coefficient $\alpha$ between the coils can be modified by using an iron piece around the coils. Figure 17a shows the relationship between the resonant frequency and the coil coupling in the case of a resonant frequency of about $1 \mathrm{kHz}$. It can be seen that the resonant frequency becomes higher with increasing coil coupling. A linear fit of the data (dashed line) yields a frequency of $1000.71 \mathrm{~Hz}$ when no mutual coupling exists between coils. The difference between the resonant frequencies for $\alpha=0.0356$ which 
corresponds to the coil coupling in the experiment of Figure 11 and for $\alpha=0$ is $1001.93-1000.71=1.22$ $\mathrm{Hz}$. The $95 \%$ confidence interval for the predicted difference is $1.22 \pm 0.08 \mathrm{~Hz}$. By adjusting the wire tension, the relationship between resonant frequency and coil coupling is studied in the case of a resonant frequency of about $1.2 \mathrm{kHz}$, as shown in Figure $17 \mathrm{~b}$. The difference between the resonant frequency for $\alpha=0.0356$ and the estimated resonant frequency for $\alpha=0$ is $1209.45-1208.35=1.10 \mathrm{~Hz}$. The $95 \%$ confidence interval for the predicted difference is $1.10 \pm 0.08 \mathrm{~Hz}$. Notice that the two frequency differences are again both more than 10 times the expected accuracy of a vibrating wire sensor.

(a)

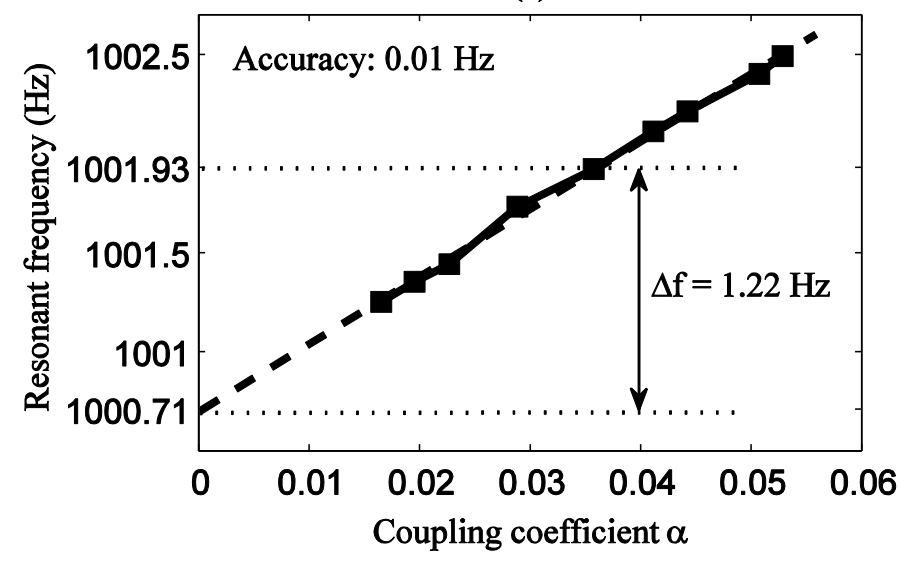

(b)

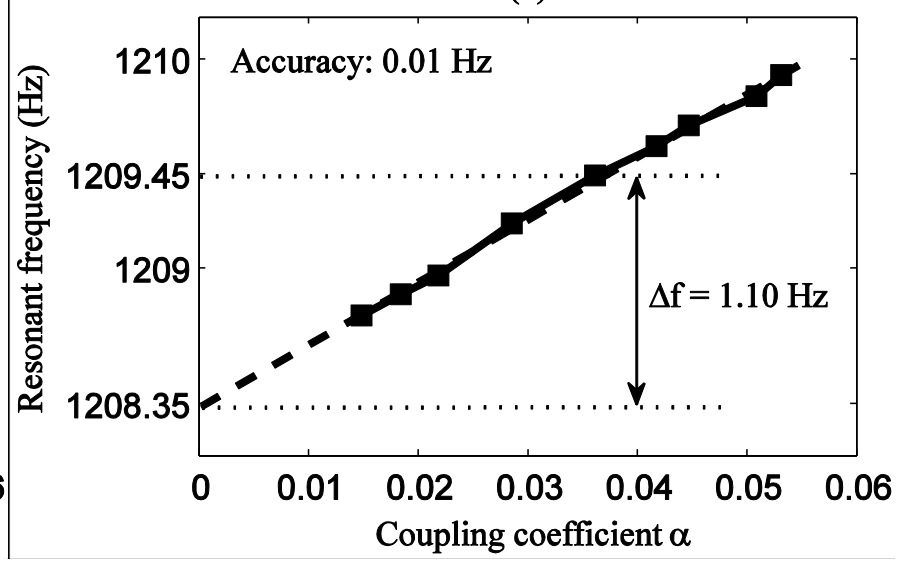

Fig. 17 Relationship between resonant frequency and coil coupling $\left(V_{\text {exc }}=1.5 \mathrm{~V}\right)$ in the case of

(a) resonant frequency of about $1 \mathrm{kHz}$ and (b) resonant frequency of about $1.2 \mathrm{kHz}$

\subsection{Analysis of experimental results}

In the case of the 1-kHz resonant frequency, in damped mode, under the condition that $V_{\text {exc }}=0 \mathrm{~V}$ and $\alpha=0$ during the measurement, the natural resonant frequency is $999.64 \mathrm{~Hz}$. Actually, in damped mode there is no magnetic force and no coil coupling during the measurement. Thus it is equivalent to a very low voltage excitation $V_{\text {exc }} \rightarrow 0$ and a very low coil coupling $\alpha \rightarrow 0$ in sustained mode. In fact, it is not possible to realize measurements in such conditions, but they can be extrapolated. Under the condition that $V_{\text {exc }}=1.5 \mathrm{~V}$ and $\alpha=0.0356$ during the measurement, the resonant frequency is $1001.93 \mathrm{~Hz}$. According to Figure $13 \mathrm{~b}$, a reduction in the excitation amplitude from $V_{\text {exc }}=1.5$ to $0 \mathrm{~V}$ would lead to a frequency variation of $\Delta f=$ $1.12 \pm 0.09 \mathrm{~Hz}$ for unchanged coil coupling $(\alpha=0.0356)$. According to Figure $17 \mathrm{a}$, a reduction in the coil coupling from $\alpha=0.0356$ to 0 would lead to a frequency variation of $\Delta f=1.22 \pm 0.08 \mathrm{~Hz}$ for unchanged excitation amplitude $\left(V_{\text {exc }}=1.5 \mathrm{~V}\right)$. Without the influences of magnetic force and coil coupling, the resonant frequency is totally reduced by $2.34 \pm 0.17 \mathrm{~Hz}$. As mentioned above, the frequency difference between the two modes corresponds to $2.29 \pm 0.22 \mathrm{~Hz}$, which is well within the $95 \%$ confidence interval of the 
prediction. In the case of the 1.2-kHz resonant frequency, the resonant frequency in damped mode is 1207.51 $\mathrm{Hz}$, whereas the resonant frequency in sustained mode under the condition that $V_{\text {exc }}=1.5 \mathrm{~V}$ and $\alpha=0.0356$ is $1209.45 \mathrm{~Hz}$. A reduction in the excitation amplitude from $V_{\text {exc }}=1.5$ to $0 \mathrm{~V}$ would lead to a frequency variation of $\Delta f=0.98 \pm 0.11 \mathrm{~Hz}$ for unchanged coil coupling (Figure 14b), and a reduction in the coil coupling from $\alpha=0.0356$ to 0 would lead to a frequency variation of $\Delta f=1.10 \pm 0.08 \mathrm{~Hz}$ for unchanged excitation amplitude (Figure 17b). Without the influences of magnetic force and coil coupling, the resonant frequency is totally reduced by $2.08 \pm 0.19 \mathrm{~Hz}$. The frequency difference between the two modes corresponds to $1.94 \pm 0.22 \mathrm{~Hz}$, which is well within the $95 \%$ confidence interval of the prediction.

\section{Conclusion}

This paper deals with the problem of the relatively important frequency difference between damped and sustained modes for vibrating wire sensors. It has been experimentally verified that the resonant frequency measured in sustained mode is shifted towards higher values because of two factors: average magnetic force that increases the wire tension and mutual coupling between coils. On the one hand, in damped mode the natural resonant frequency is independent of the magnetic force, whereas in sustained mode the resonant frequency increases with increasing magnetic force. On the other hand, the mutual coupling between the two coils only exists in sustained mode. The higher the coil coupling, the greater the increase in the resonant frequency. By removing the contribution of both magnetic force and coil coupling to the frequency difference, one recovers the damped frequency of free vibration from measurements in sustained mode. This makes it possible to use any of the two modes with confidence. For two-coil sensors, it also allows to switch from sustained mode to damped mode in the case of a single coil failure.

\section{Reference}

[1] J. M. W. Brownjohn, Structural health monitoring of civil infrastructure, Philosophical Transactions of the Royal Society of London A: Mathematical, Physical and Engineering Sciences, vol. 365, no. 1851 (2007) 589-622. doi: 10.1098/rsta.2006.1925

[2] D. M. Stefanescu, Handbook of Force Transducers: Principles and Components, Springer Science \& Business Media, New York, 2011.

[3] B. Benmokrane, M. Chekired, H. Xu, Monitoring behavior of grouted anchors using vibrating-wire gauges, Journal of geotechnical engineering 121 (1995) 466-475. doi: 10.1016/0148-9062(95)99643-c

[4] R. Shepherd, Strain measurement using vibrating-wire gages, Experimental Mechanics, vol. 4, no. 8 (1964) 244-248. doi: 10.1007/BF02322958

[5] N. Davidenkoff, The Vibrating-Wire Method of Measuring Deformation, Proceedings ASTM, vol.34, 
no.2 (1934) 847-860

[6] E. DiBiagio, A case study of vibrating-wire sensors that have vibrated continuously for 27 years, in: F. Myrvoll (Ed.), Field Measurements in Geomechanics: Proceedings of the 6th International Symposium, Oslo, Norway, 23-26 September 2003, Taylor \& Francis, London, 2003, pp. 445-458.

[7] N. Rosin-Corre, C. Noret, J.L. Bordes, L'auscultation par capteurs à corde vibrante, 80 ans de retour d'expérience, in Proc. of colloque CFBR, Chambéry, France, 2012.

[8] P. Briole, Etude théorique des appareils à corde vibrante : cas de l'extensomètre c110, comparaison des fonctionnements en amorti et en entretenu, telemac technical report, 1987.

[9] B. Mei, S. Holé, J. Lucas, Capteur à corde vibrante entretenu pérenne pour la surveillance à long terme des barrages, in Proc. 2013 Annu. Conf. Canadian Dam Association, Montréal, 2013.

[10] J. Dunnicliff, Geotechnical Instrumentation for Monitoring Field Performance, John Wiley \& Sons, Hoboken, 1993.

[11] W. Boyes, Instrumentation Reference Book, fourth ed., Butterworth-Heinemann, Oxford, 2009.

[12] K. Istvan, M. Maria, G. Bela-Zoltan, B. Szabolcs, Vibrating wire sensor measurement method by stimulation with steps of variable frequency sinusoidal pulse trains, in 2012 IEEE International Conference on Automation Quality and Testing Robotics (AQTR), 2012, pp. 587-590. doi: 10.1109/AQTR.2012.6237778

[13] T. Rossing, The Science of String Instruments, Springer Science \& Business Media, New York, 2010.

[14] F. Pelton, Guidelines for Instrumentation and Measurements for Monitoring Dam Performance, ASCE Publications, Reston, 2000.

[15] P. K. Mittal, Oscillations, Waves and Acoustics, I. K. International Pvt Ltd, New Delhi, 2010.

[16] J. R. Brauer, Magnetic Actuators and Sensors, John Wiley \& Sons, New York, 2006.

[17] B. Mei, S. Holé, J. Lucas, I. Lamarque, N. Chéron, Excitation Mode Influence on Vibrating Wire Sensor Response, Experimental Mechanics, (2015) 1-8. doi: 10.1007/s11340-015-0101-6

[18] B. Mei, S. Holé, J. Lucas, Effect of magnetic field of both excitation and measurement in vibrating wire sensors, in Proc. of the 2nd International Conf. on Technological Innovations in Nuclear Civil Engineering, Paris, 2014. 